\title{
Monitoraggio delle emissioni odorigene in un piccolo impianto di depurazione attraverso un approccio senso-strumentale
}

\author{
Federica Russo ${ }^{1}$ \\ ${ }^{1}$ Affiliation not available
}

\begin{abstract}
Le molestie olfattive indotte da impianti di trattamento di acque reflue costituiscono un vero e proprio problema ambientale. In particolare, le emissioni odorigene derivanti dall'esercizio di impianti in zone turistiche sono considerate la principale causa di disturbo notato dalla popolazione esposta. La valutazione dell'impatto indotto può essere effettuata attraverso la misurazione degli odori, utilizzando un metodo analitico, sensoriale o misto. Il sensoriale, legato al "sensore umano" è causa di notevole incertezza. L'analitico-strumentale è in grado di identificare e quantificare solo la concentrazione dei composti chimici singoli o multipli ma non l'odore complessivo di una miscela. Pertanto, integrando entrambi i metodi, nel presente articolo si vuole mostrare l'applicazione di una procedura di tipo senso-strumentale (GC/MS con porta ODP). I risultati ottenuti in uno SWWTP hanno dimostrato l'applicabilità di tale procedura nel monitoraggio delle emissioni odorigene ed hanno permesso l'identificazione di 39 sostanze diverse di cui quasi la metà responsabili di disturbi olfattivi.
\end{abstract}

\section{Problematiche delle emissioni odorigene e quadro normativo.}

Negli ultimi anni, l'attenzione dei cittadini verso la qualità dell'aria è aumentata in modo significativo e oggigiorno l'inquinamento da odori legato a diverse attività industriali, come impianti 
di trattamento e smaltimento rifiuti, è riconosciuto come un vero e proprio problema ambientale ${ }^{1}$. Nel caso specifico di piccoli impianti di depurazione (SWWTP) situati in zone turistiche, le difficoltà legate alla variabilità dei carichi concentrati in pochi mesi dell'anno possono causare molestie olfattive nell'area circostante. Quest'ultime, oltre ad essere considerate la causa principale di disturbo riscontrato dalla popolazione esposta ${ }^{2}$ hanno un impatto rilevante sull'economia del turismo ${ }^{3}$. Diversi studi hanno dimostrato che i rifiuti gassosi, oltre a causare inquinamento atmosferico sotto forma di odori, possono avere il maggiore impatto sulla popolazione in prossimità delle opere di depurazione ${ }^{4,5}$ e in alcuni casi inducono i cittadini a presentare reclami alle autorità locali ${ }^{6,7}$ mostrando sintomi associati all'odore come nausea, mal di testa e mancanza di appetito $^{8,9}$.

Per tali ragioni è importante soddisfare le esigenze della società e dei gestori degli impianti ${ }^{10}$ implementando un programma completo di gestione degli odori sotto l'aspetto della misurazione, controllo, trattamento e monitoraggio continuo ${ }^{11}$. Sfortunatamente, gli odori sono difficili da misurare. La risposta di una persona a un odore è altamente soggettiva: persone diverse trovano sgradevoli odori diversi e in concentrazioni diverse. Ciò è ulteriormente complicato dal fatto che molte emissioni odorigene, comprese quelle provenienti dagli impianti di trattamento delle acque reflue, sono costituite da molti singoli componenti odorosi e l'odore complessivo di miscele complesse non può essere facilmente misurato ${ }^{2}$. Ad oggi, infatti, la misurazione degli odori è ancora oggetto di ricerche approfondite ${ }^{1213}$ e per esso non esiste un metodo universalmente accettato.

In ambito Europeo, un passo avanti è stato fatto con la pubblicazione dello standard europeo EN13725 del 2003, in cui è stato identificato un metodo oggettivo per la determinazione della concentrazione di odore di un campione gassoso : l'Olfattometria dinamica.

In ambito nazionale, invece, la legislazione italiana non possedeva, al 2017, alcuna indicazione normativa dedicata agli odori ma solamente criteri qualitativi e riferimenti, poco dettagliati, alla 
prevenzione e mitigazione delle molestie; Con l'entrata in vigore dell'articolo 272-bis nel T.U.A., introdotto dal D. Lgs. 183/2017, e stato finalmente affrontato, seppure in una fase embrionale, il tema delle emissioni odorigene industriali e agricole ${ }^{14}$. Cio nonostante, la normativa ad oggi non possiede una regolamentazione esauriente in materia di odori a livello nazionale, ma demanda alle regioni la possibilita di indicare criteri di valutazione.

I metodi presenti allo stato dell' arte nella letteratura scientifica per la misura degli odori si raggruppano in metodi sensoriali (come l'Olfattometria), metodi analitici-strumentali (come la gascromatografia/spettrometria di massa) e metodi misti ${ }^{15}$; Le misurazioni analitiche sono state riconosciute come i metodi piu accurati per identificare e quantificare la concentrazione di composti chimici singoli o multipli. Tuttavia, queste tecniche sono costose e non consentono di avere informazioni in merito al fastidio indotto ${ }^{16}$. I metodi sensoriali, al contrario, utilizzano il naso umano come rilevatore per quantificare l'odore di una miscela nel suo insieme, indipendentemente dai singoli componenti : nel caso di aree industriali in cui le emissioni di odori sono multi sorgente l'impatto indotto puo essere valutato attraverso una combinazione di analisi olfattometrica e modelli di dispersione ${ }^{17}$. E' importante, inoltre, che le analisi sensoriali tengano conto di alcuni fattori che potrebbero influenzare la composizione dell'odore, quale la scelta del materiale di campionamento ${ }^{18}$. I sistemi misti, che sono di recente sperimentazione e applicazione nell' ambito dell'ingegneria sanitaria ambientale ${ }^{19}$, uniscono i vantaggi sia degli strumenti analitici che degli approcci sensoriali . Infatti, per eseguire il monitoraggio continuo in loco, stanno emergendo metodi sensoriali-analitici come soluzioni attraenti che consentono di superare i principali inconvenienti degli strumenti analitici ${ }^{11}$. Un esempio di metodo senso-strumentale con potenziale sviluppo futuro sono gli Instrumental Odour Monitoring Systems (IOMS) precedentemente noti anche come "Electronic nose" (e.Nose) ${ }^{20}$, oppure un sistema GC/MS con porta ODP che sara successivamente valutato attraverso un caso studio. 


\section{Presentazione del caso studio}

Il caso studio preso in considerazione per l'obiettivo preposto e quello di un impianto di trattamento delle acque reflue situato nell'Universita di Stoccarda (a sud-ovest della Germania) ${ }^{21}$. I campioni sono stati prelevati in sette punti diversi dell'impianto durante il periodo marzo-aprile 2006. Il campionamento e stato effettuato attraverso sacche di Nalophan da 3 litri e posizionate in un contenitore rigido. Successivamente, attraverso un passaggio dei campioni in una trappola polimerica porosa, sono stati adsorbiti i composti organici per poi essere desorbiti termicamente da un gascromatografo, spurgando l'adsorbente e raccogliendo i composti in una trappola di raffreddamento. L'aria umidificata, prima di essere annusata da un esperto "annusatore di odori" e stata combinata con l'effluente GC caldo. Le analisi eseguite tramite GC-MS con un odour sniffing port (ODP) e un rilevatore a ionizzazione di fiamma (FID) sono state 23 e al fine di indagare sulla relazione che vi e tra concentrazione dei principali composti odorosi e le prestazioni dell'impianto sono stati monitorati anche BOD5, COD, PH, TD, TSS e temperatura.

I risultati hanno evidenziato la presenza di circa 39 sostanze diverse all' interno della miscela volatile del GC-MS, di cui quasi la meta sono state reputate le principali responsabili dell'odore tipico di un impianto di trattamento di acque reflue. Il disolfuro di dimetile e risultato la sostanza volatile con la soglia d'odore piu bassa e piu rilevante nell' impianto, pertanto e stato definito composto "chiave". Inoltre, il maggior contributo all'impatto degli odori e risultato proveniente direttamente dalle acque reflue grezze (50\%) e subito dopo il maggiore fastidio e stato identificato dalle attivita di trattamento dei fanghi (40\%). Per quanto concerne il BOD5 e stata mostrata una buona correlazione lineare (65\%) con la concentrazione del composto chiave. Un BOD elevato produce lo sviluppo di condizioni anaerobiche nelle acque reflue ${ }^{22}$.

Questo studio ha mostrato una tecnica che combina la soggettivita sensoriale con la precisione strumentale, nonche un metodo con cui la ricerca scientifica potrebbe essere condotta sia per carat- 
terizzare l'impatto da odori sia per monitorare l'efficienza degli impianti ${ }^{21}$.

Ad oggi, sia i metodi per la misurazione di odori che le tecnologie di trattamento degli odori sono in continua sperimentazione. In un mondo in cui e crescente la preoccupazione e la salvaguardia dell' ambiente e importante, inoltre, un controllo degli odori sostenibile e rispettoso ${ }^{23}$, elemento essenziale per la mitigazione dei cambiamenti climatici e per la transizione verso un'economia decarbonizzata ${ }^{2425}$.

\section{Discussione finale}

In conclusione, le emissioni odorigene derivanti da impianti di depurazione stanno diventando una delle principali problematiche da affrontare per i gestori, al fine di evitare lamentele e potenziali impatti. Con questo caso studio, sito in Germania, si e voluto sottolineare l'importanza di una tecnica mista, senso-strumentale, nella caratterizzazione dell'impatto odorigeno in una zona turistica e la correlazione tra le concentrazioni del composto odorigeno chiave e le prestazioni dell'impianto. Un approccio integrato che combini diversi metodi e la soluzione migliore per ottenere un quadro esaustivo e completo del problema e per la definizione di una buona strategia di gestione.

Cio nonostante, va precisato che ogni situazione deve essere valutata caso per caso considerando l'applicabilita e i limiti di ciascun metodo, e soprattutto le informazioni specifiche che ciascun metodo puo fornire.

\section{References}

1.Henshaw, P., Nicell, J. \& Sikdar, A. Parameters for the assessment of odour impacts on communities. Atmospheric Environment 40, 1016-1029 (2006).

2.Gostelow, P., Parsons, S. A. \& Stuetz, R. M. Odour measurements for sewage treatment works. 
Water Research 35, 579-597 (2001).

3.Zarra, T. Procedures for detection and modelling of odours impact from sanitary environmental engineering plants.. (2007).

4.Wilson, G. E., Schroepfer, T. W. \& Huang, J. Y. C. Atmospheric Sublayer Transport and Odor Control. Journal of the Environmental Engineering Division 106, 389-401 (1980).

5.Naddeo, V. \& Korshin, G. Water energy and waste: The great European deal for the environment. Science of The Total Environment 764, 142911 (2021).

6.Aatamila, M. et al.. Odour annoyance and physical symptoms among residents living near waste treatment centres. Environmental Research 111, 164-170 (2011).

7.V.Naddeo, T.Zarra, V.Belgiorno \& S.Giuliani. Odour Impact Assessment in Industrial Areas. CHEMICAL ENGINEERING TRANSACTIONS 30, (2012).

8.Neutra, R., Lipscomb, J., Satin, K. \& Shusterman, D. Hypotheses to explain the higher symptom rates observed around hazardous waste sites.. Environmental Health Perspectives 94, 31-38 (1991).

9.Zarra, T., Naddeo, V. \& Belgiorno, V. A novel tool for estimating the odour emissions of composting plants in air pollution management. Global Nest 11, 477-486 (2009).

10.Naddeo, V., Belgiorno, V., Zarra, T. \& Scannapieco, D. Dynamic and embedded evaluation procedure for strategic environmental assessment. Land Use Policy 31, 605-612 (2013).

11.Zarra, T., Galang, M. G., Ballesteros, F., Belgiorno, V. \& Naddeo, V. Environmental odour management by artificial neural network - A review. Environment International 133, 105189 (2019).

12.Bax, C., Sironi, S. \& Capelli, L. How Can Odors Be Measured? An Overview of Methods and Their Applications. Atmosphere 11, 92 (2020). 
13.Lee, H.-D. et al.. A novel assessment of odor sources using instrumental analysis combined with resident monitoring records for an industrial area in Korea. Atmospheric Environment 74, 277-290 (2013).

14.Bruzzese, S. Valutazione dell'impatto odorigeno prodotto da un impianto di depurazione delle acque reue sulla base delle indicazioni normative esistenti. (2019).

15.Zarra, T., Giuliani, S., Naddeo, V. \& Belgiorno, V. Control of odour emission in wastewater treatment plants by direct and undirected measurement of odour emission capacity. Water Science and Technology 66, 1627-1633 (2012).

16.S.Giuliani. Strumentazione Multisensore selettiva per il monitoraggio in continuo nel settore ambientale.. (2011).

17.Naddeo, V., Zarra, T., Belgiorno, V. \& Giuliani, S. Odour Impact Assessment in Industrial Areas. CHEMICAL ENGINEERING TRANSACTIONS 30, (2012).

18.Zarra, T., Reiser, M., Naddeo, V., Belgiorno, V. \& Kranert, M. A comparative and Critical Evaluation of Different Sampling Materials in the Measurement of Odour Concentration by Dynamic Olfactometry. CHEMICAL ENGINEERING TRANSACTIONS 30, 307-312 (2012).

19.T.Zarra, V.Naddeo, M.Reiser \& M.Kranert. Odour emissions characterization from wastewater treatment plants by different measurement methods. Chemical Engineering Transactions vol.40, (2014).

20.Oliva, G. et al.. Next-generation of instrumental odour monitoring system (IOMS) for the gaseous emissions control in complex industrial plants. Chemosphere 271, 129768 (2021).

21.Zarra, T., Naddeo, V., Belgiorno, V., Reiser, M. \& Kranert, M. Odour monitoring of small wastewater treatment plant located in sensitive environment. Water Science and Technology 58, 89-94 (2008). 
22.Gostelow, P. \& Parsons, S. A. Sewage treatment works odour measurement. Water Science and Technology 41, 33-40 (2000).

23.Senatore, V. et al.. Sustainable Odour and Greenhouse Gas Emissions Control in Wastewater Treatment Plant by Advanced Biotechnology-Based System. CHEMICAL ENGINEERING TRANSACTIONS 85, 25-30 (2021).

24.Pahunang, R. R. et al.. Advances in technological control of greenhouse gas emissions from wastewater in the context of circular economy. Science of The Total Environment 792, 148479 (2021).

25.Senatore, V. et al.. Innovative membrane photobioreactor for sustainable $\mathrm{CO} 2$ capture and utilization. Chemosphere 273, 129682 (2021). 
Figure Captions

Figure 1. Rielaborazione fotografica di un impianto di depurazione e della molestia olfattiva 
Figures

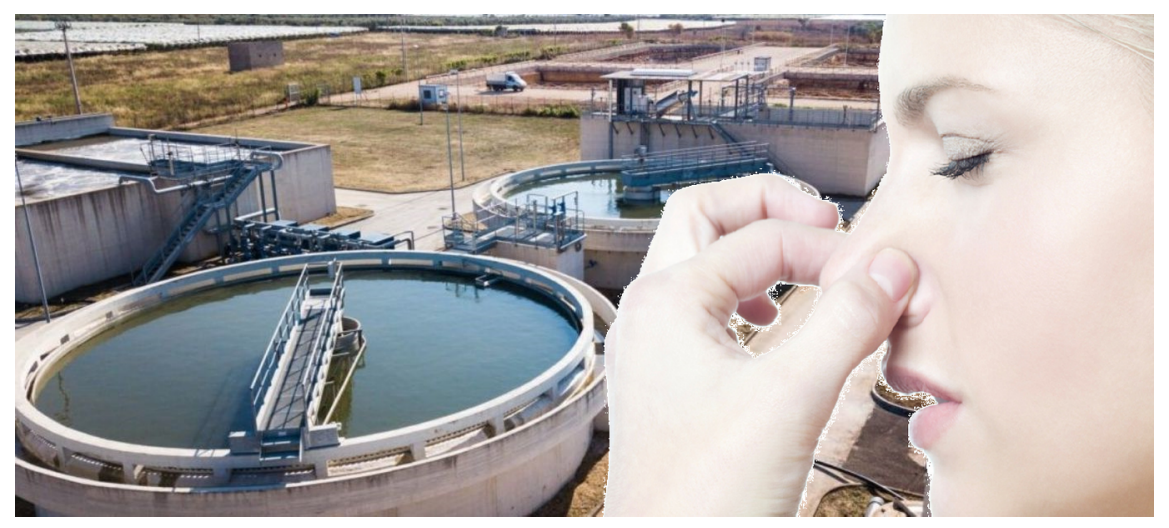

Figure 1: Rielaborazione fotografica di un impianto di depurazione e della molestia olfattiva 